\title{
Analysis of genome instability in Streptomyces ambofaciens
}

\author{
Dominique Schneider, Bertrand Aigle, Pierre Leblond, Jean-Marc Simonet $\dagger$ \\ and BERNARD DECARIS*
}

Laboratoire de Génétique et Microbiologie, Associé INRA, Faculté des Sciences de l'Université de Nancy I, BP 239, Institut de Biotechnologies, 54506 Vandoeuvre-lès-Nancy, France

(Received 8 April 1993; revised 2 June 1993; accepted 9 June 1993)

\begin{abstract}
Genetic instability in Streptomyces ambofaciens DSM 40697 is correlated with genomic instability characterized by multiple rearrangements (deletions and/or amplifications) occurring in a large unstable region. We have focused on one of the two amplifiable DNA loci which were mapped in this region: the amplifiable unit of DNA locus 6 (AUD6). The nucleotide sequence of one $A U D 6$ fragment of $1.9 \mathrm{~kb}$ reveals the presence of two open reading frames (ORF1 and ORF2) on the basis of the typical Streptomyces base composition at each of the three positions within codons. ORF1 shows some similarity with a gene encoding a regulatory protein. The presence of potential genes in this unstable locus was unexpected because deletions occurred with high frequency within this region in the genetic instability-derived mutant strains. However, transcription analyses by S1 nuclease protection experiments on the wild-type strain showed transcription of both ORF1 and ORF2. Moreover, the amplified strain reveals increased transcription of ORF1 but no transcription of ORF2. The amplification therefore results in a switch in transcription. The unstable region of $S$. ambofaciens DSM 40697 therefore is not a 'silent' region because at least some loci are transcribed.
\end{abstract}

\section{Introduction}

Genetic instability is a well-documented phenomenon in Streptomyces species, that affects characters such as sporulation, melanin formation, antibiotic production and resistance (Hütter \& Eckhardt, 1988; Leblond et al., 1990). As described below, very few markers have been localized to the genomic regions subject to genetic instability. The mutants arising from genetic instability have been shown to carry large chromosomal deletions (Birch et al., 1989; Demuyter et al., 1991; Leblond et al., 1991). In addition to deletions, amplified DNA sequences are found with high frequencies in such mutant strains (Cullum et al., 1986; Hütter \& Eckhardt, 1988). The nomenclature proposed elsewhere (Fishman \& Hershberger, 1983) is used: ADS for amplified DNA sequence, and AUD for amplifiable unit of DNA.

\footnotetext{
*Author for correspondence. Tel. +3383912096 ; fax +338391 2500 .

†Present address: Université de Paris-Sud, CNRS-URA 1354, Bât. 400, 91405 Orsay Cedex, France.

Abbreviations: AUD, amplifiable unit of DNA; ADS, amplified DNA sequence; nt, nucleotide; wt, wild-type.

The nucleotide sequence data reported in this paper have been submitted to the EMBL Data Library and have been assigned the accession number X70975.
}

Streptomyces ambofaciens DSM 40697 exhibits two levels of genetic instability: (i) a basic one; and (ii) hypervariability, another aspect of this phenomenon which is related to DNA amplification (Leblond et al., 1989). Deletions have been characterized in all mutant strains and amplifications in about $17 \%$ of all mutant strains (Leblond et al., 1991). All amplified strains of $S$. ambofaciens carry ADSs belonging to two amplifiable loci: $A U D 6$ and $A U D 90$. Pulsed-field gel electrophoresis experiments have shown that these two AUD families are located within the same chromosomal region leading to the notion of the 'unstable region' in the S. ambofaciens genome (Leblond et al., 1991). Furthermore, the ADSs are associated with deletions which remove part of the proximal copy(ies) of these ADSs (Demuyter et al., 1991). AUD6 constitutes a rearrangement hotspot (Demuyter et al., 1991). Indeed, 20\% of the unamplified mutant strains present a deletion endpoint in AUD6. Furthermore, $13 \%$ of the amplified strains carry an ADS belonging to this locus which could therefore contain a deletion and/or amplification signal.

The $A U D$-encoded functions remain unknown in most Streptomyces species although it has been shown (Dittrich et al., 1991) that a chloramphenicol resistance determinant localized in an amplifiable and deletable region of $S$. lividans 1326 is predicted to encode a putative transmembrane protein. Furthermore, the 'un- 
stable region' of the $S$. glaucescens chromosome contains the melC and strS genes (Birch et al., 1989). Several examples of antibiotic resistance genes are known which are located within AUDs (Potekhin \& Danilenko, 1985; Hornemann et al., 1987), as is a mercury resistance determinant (Altenbuchner \& Brüderlein, 1986). In $S$. lividans, genetic instability seems to be a two-stage process in which the initial chloramphenicol sensitivity mutation switches on a dramatic arginine requirement instability (Altenbuchner \& Cullum, 1984; Flett \& Cullum, 1987). The arginine gene $\arg G$ is affected by this genetic instability. This gene was mapped to the 30 'clock region of the $S$. coelicolor A3(2) genome (Kieser et al., 1992) in which very few markers were localized by classical genetic analyses and which is thought to be a 'silent' region. Comparison between the physical map of S. lividans (Leblond et al., 1993) and the physical and genetic map of $S$. coelicolor has shown that the $S$. lividans unstable region corresponds to the silent region of S. coelicolor mapped at 3 o'clock.

In this paper, we report the study of $A U D 6$, which is a rearrangement hotspot in S. ambofaciens DSM 40697. The nucleotide sequence analysis of one fragment of AUD6 reveals the presence of two open reading frames (ORFs). The two ORFs are transcribed in the wild-type (wt) strain and one of them shows increased transcription in the amplified strain.

\section{Methods}

Bacterial strains and plasmids. S. ambofaciens DSM 40697 (Hütter, 1967) was used as the wt strain. The amplified mutant strain NSA6 (Demuyter et al., 1991), which is amplified for AUD6, was a spontaneous derivative of the wt strain. In the progeny of NSA6 a deamplified mutant strain (called NSA1641) was isolated whose deletion includes the $1.9 \mathrm{~kb}$ BamHI fragment of $A U D 6$.

Escherichia coli HB101 (Boyer \& Roulland-Dussoix, 1969) and Escherichia coli SURE (Stratagene) (uvrC umuC recB hsdR mcr A mcrB $m r r$ endA) were the hosts for cloning. Plasmid pBluescript KS (Short $e t$ al., 1988) and cosmid pHC79 (Hohn \& Collins, 1980) were used.

Media and culture conditions. Streptomyces strains were grown at $37^{\circ} \mathrm{C}$ on plates of Hickey-Tresner (HT) medium (Pridham et al., $1956 / 57$ ). Large-scale DNA isolation was carried out after growth for $48 \mathrm{~h}$ at $37^{\circ} \mathrm{C}$ with shaking (200 r.p.m.) in YEME liquid medium (Hopwood et al., 1985) supplemented with glycine (0.25\%). E. coli strains were grown at $37^{\circ} \mathrm{C}$ in LB liquid medium (Maniatis et al., 1982). For growth curves and RNA isolations, JLP5 medium (J. L. Pernodet, unpublished results) (yeast extract, $0.7 \% ; \mathrm{NaCl}, 0.5 \%$; $\mathrm{NaNO}_{3}, 0.1 \%$; MOPS, $2.1 \%$, w/v; glycerol, $3.6 \%$, v/v; pH 7.5) was inoculated with $5 \times 10^{7}$ c.f.u. after growth for $2 \mathrm{~h}$ at $37^{\circ} \mathrm{C}$ in pregermination medium (casamino acids, $1 \%, \mathrm{w} / \mathrm{v}$; yeast extract, $1 \%$, $\mathrm{w} / \mathrm{v})$. The cultures were then incubated at $25^{\circ} \mathrm{C}$ with shaking $(200$ r.p.m.). At each time point where RNA was prepared, DNA was also extracted and hybridization experiments revealed the same results as for the cultures at $37^{\circ} \mathrm{C}$. Furthermore, RNA isolations were performed after culturing at $25^{\circ} \mathrm{C}$ because all studies in our laboratory on gene expression in the unstable region were done at this temperature (Dary and others, unpublished results).
Growth curves. Growth curves of the wt and mutant strains were obtained by following the DNA content of these strains. Samples $(1 \mathrm{ml})$ were harvested from the cultures at different times. The mycelia were then sonicated using a Labsonic $L$ apparatus (B. Braun), centrifuged and resuspended in $1 \times \operatorname{SSC}(0.15 \mathrm{M}$-sodium chloride, $0.015 \mathrm{M}$-sodium citrate). Colorimetric estimation of DNA was carried out using the diphenylamine reaction (Burton, 1956), with salmon sperm DNA as standard.

Isolation of total DNA and restriction analyses. DNA extraction and purification were performed as described by Demuyter et al. (1988). Restriction endonucleases were purchased from Boerhinger Mannheim and used according to the manufacturer's recommendations. DNA fragments were electrophoresed on $0.8 \%$ agarose gels as described (Maniatis et al., 1982). Bacteriophage $\lambda$ DNA digested with HindIII was used as a size standard (Daniels et al., 1983).

RNA isolation and manipulation. RNA was extracted from liquid cultures. RNA isolation and low-resolution S1 nuclease protection experiments were performed as described by Hopwood et al. (1985). Forty micrograms of RNA were used in these experiments. The probe was the AUD6 $1.9 \mathrm{~kb} \mathrm{BamHI}$ fragment shown in Fig. 1. Bands on autoradiographs were quantified by densitometric analyses. RNA isolations were performed at different growth stages. The growth curves, RNA isolations and low-resolution S1 nuclease experiments were done at least three times for each sample.

${ }^{32} P$ labelling of DNA, Southern blotting and hybridizations. DNA probes were ${ }^{32}$ P-labelled (Amersham) as described by Feinberg \&

(a)

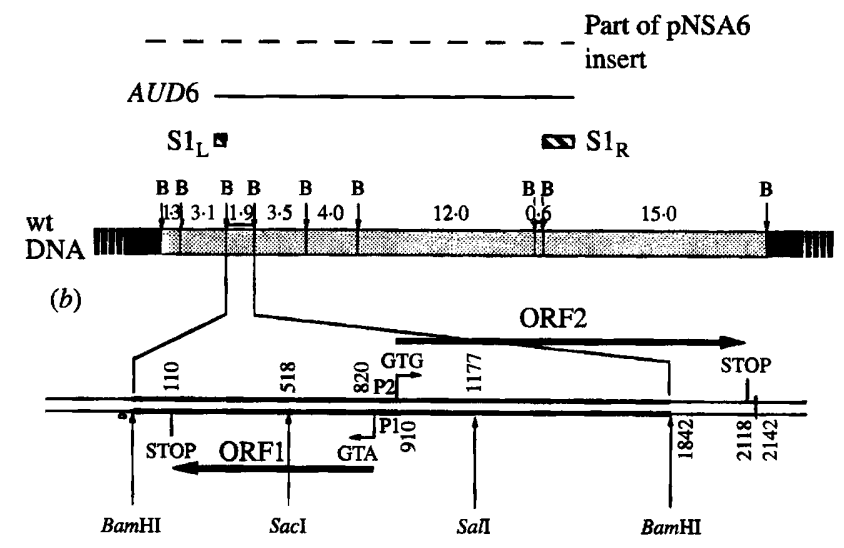

Fig. 1. BamHI restriction map of the $S$. ambofaciens DSM 40697 $A U D 6$. (a) S1 corresponds to the extremities of $A U D 6$ and is the fragment generated by the reiteration of $A U D 6 . \mathrm{S1}_{\mathrm{L}}$ and $\mathrm{S1}_{\mathrm{R}}$ are the left and the right ends of $S 1$, respectively (Demuyter et al., 1991). The $1.9 \mathrm{~kb} \mathrm{BamHI}$ fragment is overlined. Restriction fragment sizes are given in kb. AUD6 (solid line over the restriction map) and part of the $40 \mathrm{~kb}$ insert in cosmid pNSA6 (dotted line) are shown. B, BamHI. (b) Schematic representation of the $1.9 \mathrm{~kb} \mathrm{BamHI}$ fragment sequence and orientation of this fragment within AUD6 (at the top). The thick black arrows show the direction of transcription for both ORFs. The putative initiation and termination codons of ORF1 and ORF2 are shown. P1 and $P 2$ are, respectively, the putative ORF1 and ORF2 promoters. The nucleotide positions are given in bp. The ORF2 stop codon is located in the $3.5 \mathrm{~kb}$ Bam HI fragment and the sequencing strategy is the same as that employed for the $1.9 \mathrm{~kb} B a m \mathrm{HI}$ fragment. The two probes specific for each ORF used for the transcription analyses are delimited by their restriction sites: BamHI-SacI (positions 0-518) and SalIBamHI (positions 1177-1842). 
Vogelstein (1983) using a nick-translation kit (Amersham) or a Megaprime kit (Amersham).

Southern blotting (Southern, 1975) was carried out by the capillary transfer method with the Vacugene system (LKB) onto Hybond-N membranes (Amersham). The DNA was depurinated with $0.25 \mathrm{M}-\mathrm{HCl}$ for $30 \mathrm{~min}$ before denaturation (in $1.5 \mathrm{M}-\mathrm{NaCl}, 0.5 \mathrm{M}-\mathrm{NaOH}$ for $30 \mathrm{~min}$ ) and neutralization (in $1.5 \mathrm{M}$-Tris $/ \mathrm{HCl}, 1.5 \mathrm{M}-\mathrm{NaCl}$ for $30 \mathrm{~min}$ ) and vacuum-transferred with $20 \times$ SSC for $90 \mathrm{~min}$. Prehybridization, hybridization and washing conditions have been described previously (Demuyter et al., 1988).

Construction of a genomic library of S. ambofaciens DSM 40697. Total DNA from $S$. ambofaciens DSM 40697 was partially digested with PstI to generate fragments of $35-40 \mathrm{~kb}$. These were ligated into PstI-digested pHC79, which was then packaged by using the $\lambda$ DNA in vitro packaging kit (Amersham). The cosmids were introduced into E. coli HB101 strain and recombinant clones were selected by in situ hybridization.

DNA sequencing. DNA sequences were determined by the dideoxychain termination method (Sanger et al., 1977) with pBluescript KS as vector. They were carried out with the TaqTrack sequencing system (Promega), using Taq DNA polymerase (Innis et al., 1988) for chain elongation. The nucleotide analogue 7-deaza-dGTP was substituted for dGTP in the reaction mixtures. Products of the reactions were analysed on $5 \%(\mathrm{w} / \mathrm{v})$ polyacrylamide/7 M-urea gels. Nested deletions were carried out within the sequenced fragment using the double-stranded nested deletion kit (Pharmacia). Several parts of the $1.9 \mathrm{~kb}$ BamHI fragment were subcloned in different pBluescript KS restriction sites. Three primers were used: the T3 and SK primers (USBC) and the M13 forward primer (Promega) of pBluescript KS. Both strands were sequenced independently.

\section{Results}

\section{Cloning of AUD6}

It has been shown (Demuyter et al., 1991) that $A U D 6$ is a deletion hotspot and amplifiable locus in $S$. ambofaciens DSM 40697. To study AUD6 structure, a genomic library of $S$. ambofaciens DSM 40697 was constructed in pHC79 and in situ hybridization experiments using the cloned extremities of $A U D 6$ (probe S1) (Demuyter et al., 1991) revealed one positive clone, containing a recombinant cosmid called pNSA6. This $40 \mathrm{~kb}$-cosmid contained a part of AUD6 (Fig. 1). The AUD6 $1.9 \mathrm{~kb} B a m \mathrm{HI}$ fragment was subcloned from pNSA6 (using $E$. coli SURE as host strain and pBluescript KS as cloning vector) to generate recombinant plasmid pNSA61. Hybridization experiments, using pNSA6 as a probe, revealed strong similarities with two other $S$. ambofaciens isolates: ATCC 23877 and ETH 11317 (data not shown), indicating the presence of this unstable region. The $1.9 \mathrm{~kb}$ fragment was present in these strains indicating that all the characteristics of this fragment are likely to be present in the ATCC and ETH isolates.

It has been shown previously (Demuyter et al., 1991) that amplification in the NSA6 strain is associated with a deletion which removes part of the proximal copy(ies) of the ADS. Indeed, hybridization experiments using pNSA61 as a probe revealed faint signals in the NSA6- amplified strain (data not shown) which were absent from strains DSM 40697 and NSA1641 (see Methods). These signals could thus correspond to a junction fragment produced as a result of the deletion. Thus, the right deletion endpoint in this strain could be localized within the $1.9 \mathrm{~kb} \mathrm{BamHI}$ fragment. This fragment could therefore contain sequences involved in the amplification and/or deletion processes in S. ambofaciens.

\section{Nucleotide sequencing of the $1.9 \mathrm{~kb}$ BamHI fragment of AUD6}

The nucleotide sequence of the BamHI fragment is given in Fig. 2(a) and extends from nucleotides (nt) 1 to 1842 ( $\mathrm{G}+\mathrm{C}$ composition of $69 \%$ in good agreement with the high $\mathrm{G}+\mathrm{C}$ content of the Streptomyces genome: 70 $74 \%$ ). Nucleotides $1843-2142$ correspond to the beginning of the adjacent $3.5 \mathrm{~kb} \mathrm{BamHI}$ fragment sequence (Fig. 1a).

The sequence contained two ORFs which showed base composition at each of the three positions within codons typical of that found for Streptomyces species (Bibb et al., 1984; Hopwood et al., 1986). Fig. 1(b) shows the localization of these two ORFs, which were on the opposite strands from each other, and the orientation of the $1.9 \mathrm{~kb} B a m \mathrm{HI}$ fragment in $A U D 6$. ORF1 (nt $820-110$ ) had a codon base composition of about 75, 43 and $90 \% \mathrm{G}+\mathrm{C}$ for the first, second and third positions of the codons, respectively, and began with ATG. This ORF potentially encodes a protein of 237 amino acids (Fig. 2a). In addition, there is a sequence (P1) resembling the Streptomyces consensus promoter sequence (Hopwood et al., 1986) immediately upstream of this ORF (Fig. $2 b$ ). No conventional ribosome-binding site was detected upstream of the ORF1 translational initiation codon. Although of rare occurrence, similar results were obtained for the aph gene of Streptomyces fradiae and for the ermE gene of Saccharopolyspora erythraea (Streptomyces erythraeus) (Bibb et al., 1985). Perhaps, this is a feature of the translational initiation of many Streptomyces genes.

ORF2 (nt 910-2118) overlapped the $1.9 \mathrm{~kb}$ BamHI fragment and the adjacent AUD6 $3.5 \mathrm{~kb} B a m \mathrm{HI}$ fragment. This ORF was 1209 nucleotides long and presented a base composition of about 70,46 and $95 \% \mathrm{G}+\mathrm{C}$ for the first, second and third positions within codons, respectively. This ORF was on the opposite strand from the first one. It began with GTG. Several nucleotides upstream of this GTG, a potential ribosome-binding site (AGGAG) was present. ORF2 potentially encodes a protein of 403 amino acids. A sequence (P2) resembling the consensus promoter sequence of Streptomyces was found upstream of this ORF (Fig. $2 b$ ).

The putative promoters $\mathrm{P} 1$ and $\mathrm{P} 2$ were located 
(a)

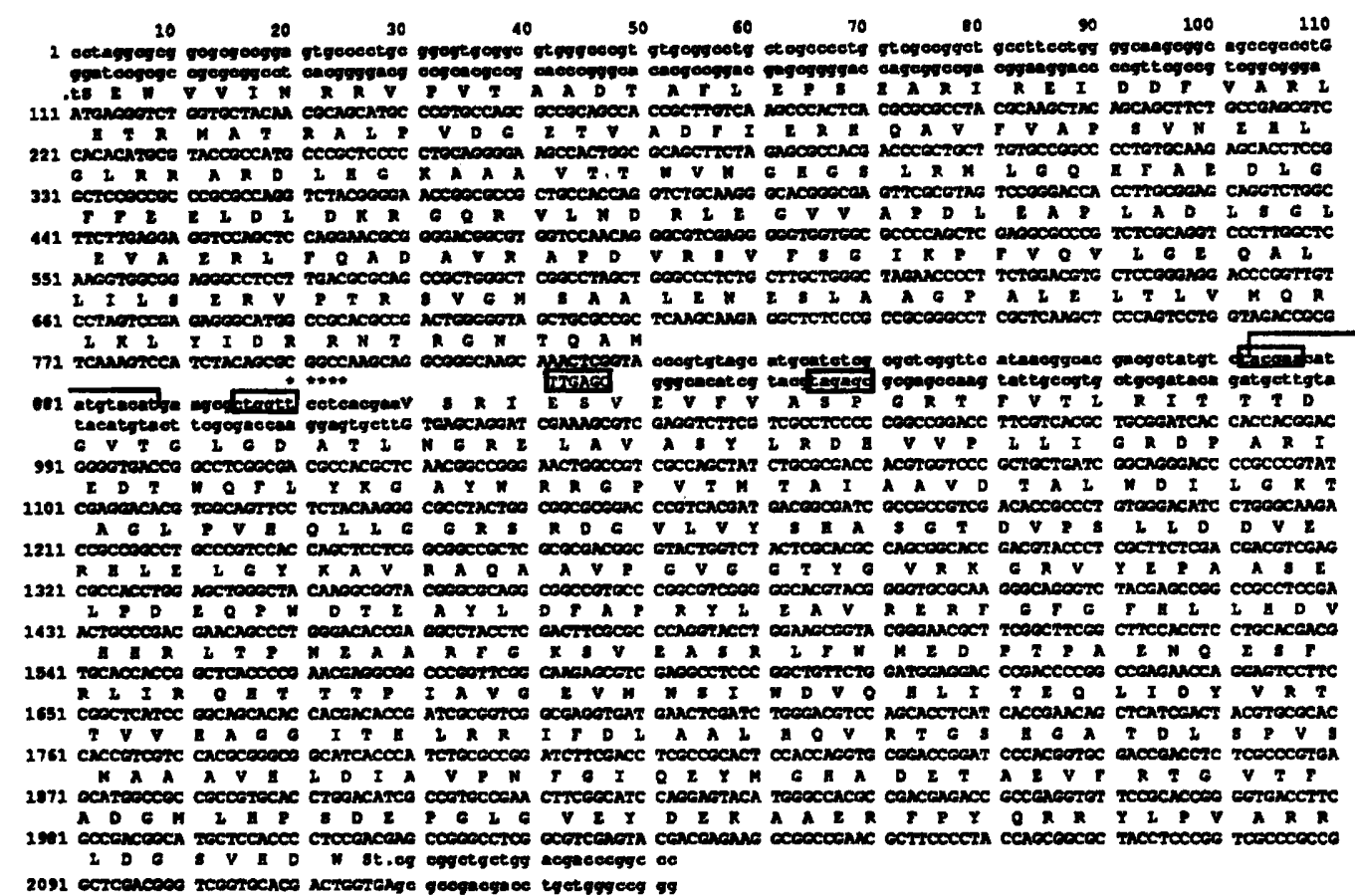

(b)

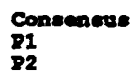

Tranos - $18 \mathrm{bp}$ - tacgar rregto - 17 bp - eAcoer (c)

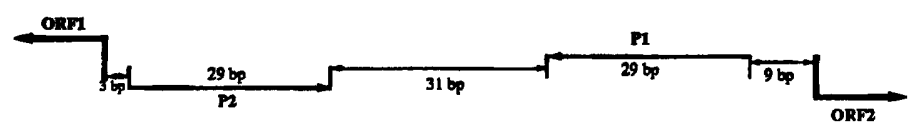

Fig. 2. Nucleotide sequence of the AUD6 $1.9 \mathrm{~kb}$ Bam HI fragment and $300 \mathrm{bp}$ part of the adjacent $3.5 \mathrm{~kb}$ Bam HI fragment. (a) Nucleotide sequence. Nucleotides 1-1842 corresponded to the $1.9 \mathrm{~kb}$ Bam HI fragment sequence. Nucleotides 1843-2142 corresponded to the beginning of the adjacent $3.5 \mathrm{~kb}$ BamHI fragment sequence. The coding strand for each ORF, and both strands for the intergenic region and for the putative promoters are shown. Upper case letters correspond to the ORF1 and ORF2 sequences; lower case letters correspond to sequences outside of both ORFs. The amino acid sequence of ORF1 (nt 820-110) and ORF2 (nt 910-2118) is shown over their nucleotide sequence. The ORF2 putative ribosome-binding site is highlighted with asterisks. The ORF1 putative promoter P1 is boxed with thin lines (top strand), whereas the ORF2 putative promoter P2 is boxed with bold lines (bottom strand). The sequence resembling the gnt $R$ gene operator of the $B$. subtilis gluconate operon is overlined with a bracket. The arrows at the left represent the direction of transcription of both ORFs. Stop codons are indicated by St. (b) Comparison of the putative promoters located upstream of ORF1 (P1, middle line) and ORF2 (P2, bottom line) with the consensus sequence (top line) established for Streptomyces (Hopwood et al., 1986). Upper case letters represent the most conserved nucleotides in the consensus sequence. (c) Promoter region of ORF1 and ORF2. $\mathrm{P} 1$ is the ORF1 promoter and $\mathrm{P} 2$ is the ORF2 promoter.

between ORFs 1 and 2 (Fig. 2). Moreover, they were face-to-face. Transcription of ORF1 and ORF2 was divergent from these two putative face-to-face promoters. In the regions of divergent transcription with face-to-face promoters, the distances between the translational start sites range from 64 to 235 bp (Beck \& Warren, 1988). The two translational start sites for AUD6 ORF1 and ORF2 were $89 \mathrm{bp}$ apart and this region of DNA revealed an A+T content of $47 \%$, which is high for Streptomyces.

\section{$O R F$ analyses}

The amino acid sequences of both ORFs were compared with the NBRF protein sequence database using the FASTA program (Lipman \& Pearson, 1985). The ORF1 predicted protein sequence revealed a $23.8 \%$ similarity (over 227 amino acids) with the Bacillus subtilis GntR protein (Fujita \& Fujita, 1987), which is the repressor of the gluconate operon (Fig. $3 a$ ). Furthermore, analysis of the intergenic region revealed the presence of a sequence (located upstream of the translation initiation codon) which presented a strong similarity (14/16 nucleotides) with the operator region recognized by GntR in B. subtilis which is located upstream of the initiation codon of the gnt $R$ gene (Fujita \& Miwa, 1989) (Fig. 3b). This region showed an imperfect dyad symmetry (Fig. $3 b$ ).

The ORF1 putative protein showed some features of DNA-binding proteins such as helix-turn-helix domains, one of which could be a good candidate for DNA binding, and which revealed a good alignment (with the FASTA program) with the predicted binding domains of GylR, the repressor of the $S$. coelicolor glycerol operon (Smith \& Chater, 1988), AsnC (an activator of as- 
(a)

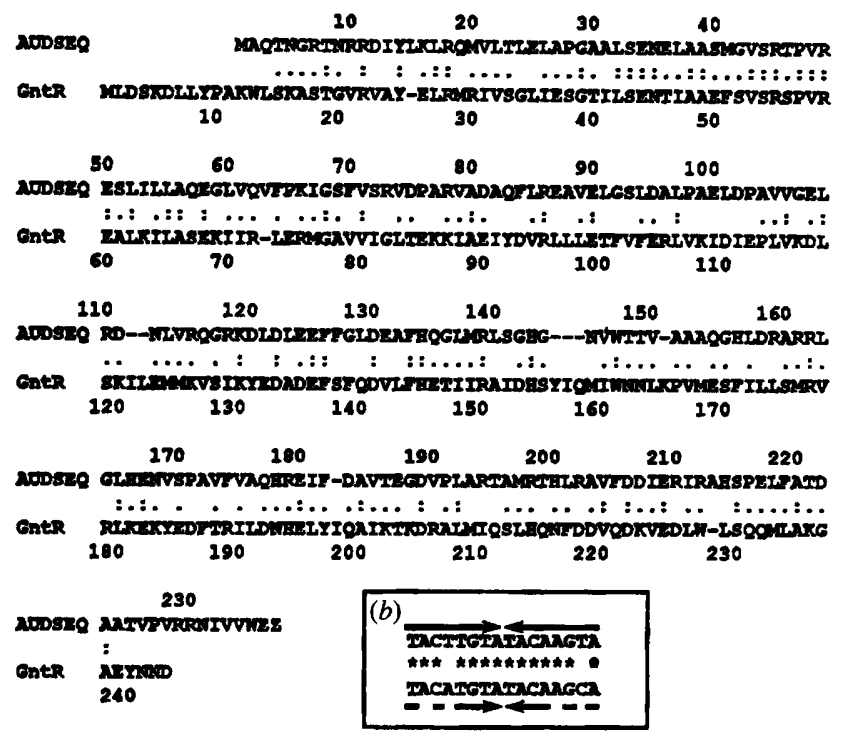

Fig. 3. Analysis of the predicted $S$. ambofaciens AUD6 ORF1 protein. (a) Comparison of the predicted protein (AUDSEQ) with the B. subtilis $g n t R$ gene product (GntR). Colons represent identical amino acids, whereas points represent conservative changes as defined by Lipman \& Pearson (1985). (b) Comparison of the operator sequence recognized by GntR (Fujita \& Miwa, 1989) (top line) with the corresponding sequence of $A U D 6$ (bottom line and overlined in Fig. 2). The arrows indicate the dyad symmetry. The stars indicate the conserved nucleotides.

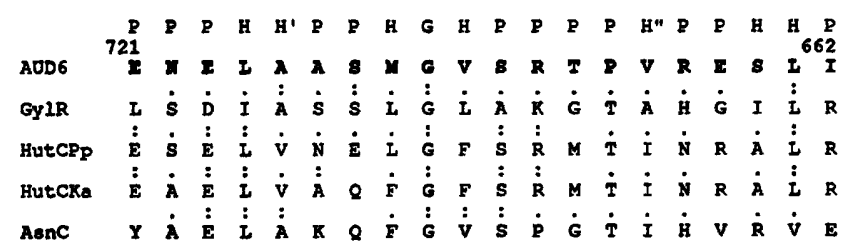

Fig. 4. Comparison of the putative helix-turn-helix motif of the predicted AUD6 protein with predicted helix-turn-helix motifs of different regulatory proteins. Colons and points are defined in the legend to Fig. 3. AUD6 is the sequence of the putative helix-turn-helix domain of the AUD6 ORF1. The position of this domain in the nucleotide sequence (Fig. 2) is indicated. GylR is the predicted sequence of the helix-turn-helix domain of the glycerol operon repressor of $S$. coelicolor (Smith \& Chater, 1988). HutCPp and HutCKa are the predicted sequences of the helix-turn-helix domains of the histidine operon repressors of Pseudomonas putida (Allison \& Phillips, 1990) and of Klebsiella aerogenes (Schwacha \& Bender, 1990), respectively. AsnC is the transcriptional activator of asnA in E. coli (Dodd \& Egan, 1987; Kölling \& Lother, 1985). The consensus residue types at each position of a typical helix-turn-helix DNA-binding domain (Pabo \& Sauer, 1984) are indicated in the top line. G, predominantly glycine; $\mathrm{H}$, usually non-polar; $\mathrm{H}^{\prime}$, usually non-polar (alanine favoured); $\mathrm{H}^{\prime \prime}$, nonpolar (usually valine or isoleucine); $\mathbf{P}$, usually polar.

paragine synthetase A transcription in E. coli; Dodd \& Egan, 1987; Kölling \& Lother, 1985), HutC (the repressor for the histidine utilization genes) of Pseudomonas putida (Allison \& Phillips, 1990) and of Klebsiella aerogenes (Schwacha \& Bender, 1990) (Fig. 4). The

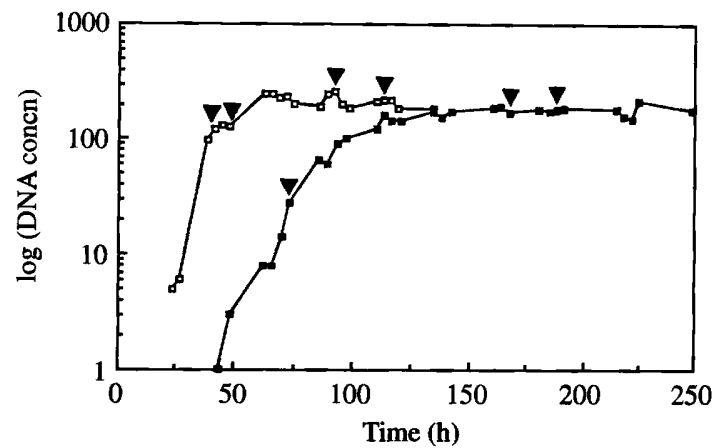

Fig. 5. Growth curves for the wt ( $\square$ ) and NSA6 ( $\square$ ) strains. The curves were obtained by following the DNA concentration during growth (see Methods). The black arrowheads indicate the time at which the RNA extractions were prepared.

corresponding region of the AUD6 ORF1 sequence also fulfilled the general criteria for forming the helix-turnhelix structure characteristic of many DNA-binding domains (Pabo \& Sauer, 1984). These results are consistent with the proposal that the potential protein encoded by ORF 1 could act as a regulatory protein. No homology with known sequences was found for the ORF2 predicted protein sequence.

\section{Transcriptional analysis}

Low-resolution S1 nuclease mapping was performed with RNA from the wt and the amplified NSA6 strains with the AUD6 $1.9 \mathrm{~kb}$ Bam HI fragment as the protected DNA. The negative control consisted of the NSA1641 strain in which the deletion included the $1.9 \mathrm{~kb}$ BamHI fragment.

With this $1.9 \mathrm{~kb}$ fragment probe, the transcript corresponding to ORF1 was expected to have a size of about 800-850 nt (taking into account the potential ORF1 promoter), and the ORF2 potential transcript was expected to have an overlap of about $1000 \mathrm{nt}$ with the probe.

After hybridization of the unlabelled DNA probe with RNA isolated from different strains and S1 nuclease treatment, the potential DNA-RNA hybrids were subjected to alkaline electrophoresis and the remaining DNA strand was transferred onto nylon membranes. Hybridization using radioactive probes specific to ORF1 and ORF2, respectively, allowed us to determine the transcription of both ORFs. The ORF1- and ORF2specific probes were, respectively, the BamHI-SacI fragment and the BamHI-SalI fragment of the $1.9 \mathrm{~kb}$ BamHI fragment (Fig. 1b).

RNA was extracted from wt and NSA6 at different stages of growth (Fig. 5). Extractions were realized three times for each strain and time point and the results were reproducible. NSA1641 gave a curve similar to that of NSA6. These two mutant strains grew differently from 


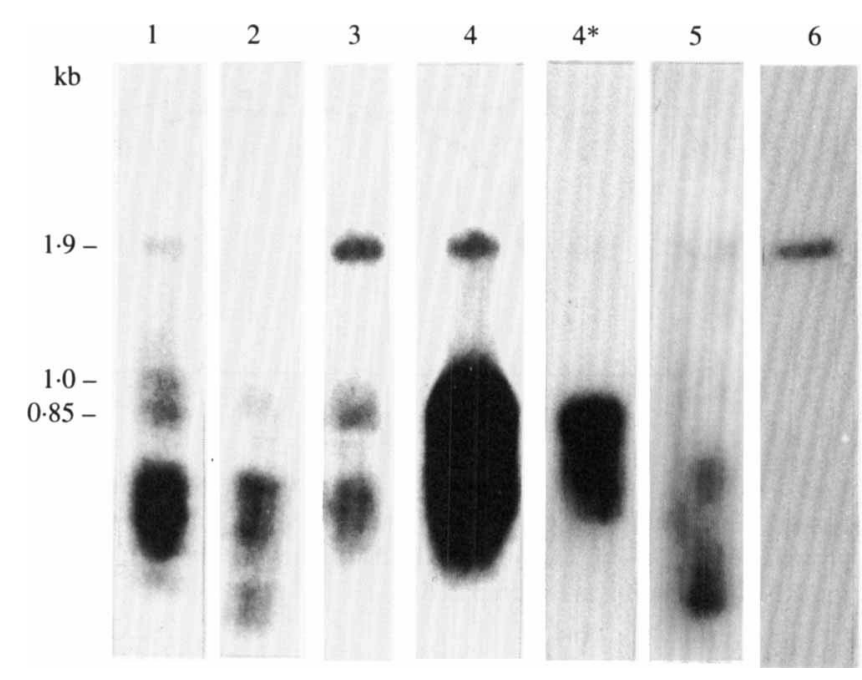

Fig. 6. Low-resolution S1 nuclease mapping of ORF1 and ORF2 mRNA. The protected DNA is the $1.9 \mathrm{~kb}$ BamHI fragment. After transfer of the DNA counterpart of the DNA-RNA hybrids onto nylon membranes, a probe specific to ORF2 (lanes 1 and 2) or to ORF1 (lanes 3, 4 and $4^{*}$ ) was used. Each probe gave the same pattern for lanes 5 and 6. Lanes: 1 , wt $S$. ambofaciens DSM 40697 mRNA (stationary phase); 2, NSA6 mRNA (stationary phase); 3, wt $S$. ambofaciens DSM 40697 mRNA (stationary phase); 4, NSA6 mRNA (stationary phase); 4*, NSA6 mRNA (stationary phase); 5, NSA1641 mRNA (stationary phase); $6,1.9 \mathrm{~kb}$ BamHI $A U D 6$ fragment. The exposure time was $4 \mathrm{~d}$ with two intensifying screens for all lanes except lane $4^{*}$ which was exposed for $1.5 \mathrm{~d}$ without an intensifying screen. The $1.9 \mathrm{~kb}$ signal present in lanes $1,3,4,4^{*}$ and 5 corresponded to the DNA-DNA probe renaturation. The lower molecular mass signals appeared also in the negative control (lane 5). Thus, these signals could be caused by nonspecific hybridization. The patterns obtained with RNA extracted from exponential growth cultures were the same as that of lane 5. Lane $4^{*}$ shows that the increased transcription observed in NSA6 corresponds to ORF1.

the wt strain and revealed a delayed growth compared to the wt strain. Furthermore, two other independently isolated $A U D 6$ mutant strains showed the same delayed growth, whereas one $A U D 90$ mutant strain without any rearrangement in AUD6 presented the same growth curve as the wt strain (data not shown). This delayed growth seemed to be a characteristic of mutant strains harbouring deletions in AUD6.

Fig. 6 shows the results of S1 nuclease protection experiments. With the ORF2-specific probe, two signals at about 1000 and $850 \mathrm{nt}$ were visualized for both RNA preparations from the wt strain during stationary phase (Fig. 6, lane 1), but none for RNA extracted during exponential growth. Gel electrophoresis of RNA samples from exponential phase showed that the relevant RNA preparations were of suitable quality (due to the visualization of the rRNA bands and to the absence of smear). Thus, the absence of signals during exponential growth indicated an absence of transcription. Thus, ORF2 was transcribed during the stationary phase in the wt strain. In these experiments, the detected signals are the DNA corresponding to the overlap between RNA and DNA in the heteroduplex exposed to S1 nuclease. The $1000 \mathrm{nt}$ transcript might correspond to that predicted from the ORF2 organization shown in Fig. 1(b) and from the DNA probe used in the $\mathrm{S} 1$ nuclease protection experiment (the $1.9 \mathrm{~kb}$ Bam HI fragment). Two initiation sites for ORF2 transcription might exist, which could explain the two signals. Furthermore, another GTG translation start codon (in the same phase as the first one) was localized at nucleotide position 994 (Fig. 2). These two signals were undetected in the amplified strain (Fig. 6, lane 2) indicating that ORF2 transcription was abolished in this strain.

The ORF1-specific probe revealed a signal at about $850 \mathrm{nt}$ in both RNA samples isolated during stationary phase from the wt strain (Fig. 6, lane 3). No signal was detected with the sample isolated during exponential growth. The transcript size of about $850 \mathrm{nt}$ was in good agreement with that predicted from the nucleotide sequence. This transcript could not be the same as that of the ORF2 of the same size because there was insufficient homology (on the basis of the nucleotide sequence) between ORF1 and ORF2. Furthermore, the two ORFspecific probes did not give the same results in the amplified strain (see below). The same ORF1 probe used in the amplified strain revealed the same signal as that in the wt strain but it was much stronger (Fig. 6, lane 4). Thus, the amplified strain showed increased transcription of ORF1. Indeed, densitometric analyses revealed that the hybridization signal in the amplified strain was about 100 -fold that in the wt strain. The amplified strain possessed an ADS copy number of 90 . Thus, it seems that this increase in intensity corresponds to increased transcription associated with the amplification level. Increased transcription was observed for both RNA extractions prepared in stationary phase for strain NSA6. No transcription was observed for ORF1 in NSA6 during exponential growth. All the RNA samples (for both the wt and NSA6 strains) extracted during exponential growth showed a pattern similar to the one presented in Fig. 6, lane 5. Lane 5 shows the control with RNA not homologous to the probe (indeed, the genomic DNA of NSA1641 revealed no hybridization with the $1.9 \mathrm{~kb}$ BamHI fragment).

The $1.9 \mathrm{~kb}$ signal, still present in Fig. 6, lane 5, corresponded to the DNA-DNA probe renaturation.

In conclusion, amplification of $A U D 6$ might induce a switch in the transcription of both ORFs.

\section{Discussion}

The sequence of the $1.9 \mathrm{~kb}$ BamHI fragment, which contained a deletion end-point in strain NSA6, revealed the presence of two ORFs on the basis of the 
Streptomyces codon usage, which presents a bias due to the high $\mathrm{G}+\mathrm{C}$ content of the Streptomyces genome (Wright \& Bibb, 1992). The degree of synonymous codon usage (SCU) bias of a gene is positively correlated with its level of expression. The SCU of ORF1 and ORF2 were similar to those of the 64 Streptomyces genes studied by Wright \& Bibb (1992), strongly indicating that both ORFs might be expressed. Computer analyses of the ORF1 product (homologies with the gluconate operon repressor of $B$. subtilis, presence of a putative helix-turn-helix motif) lead us to propose that ORF1 encodes a regulatory DNA-binding protein.

A sequence showing similarity to the consensus promoter sequence of Streptomyces was present upstream of the translation initiation codon of each ORF. The ORF2 promoter did not contain the $T$ residue at the sixth position of the ' -10 ' box but three out of the six nucleotides in this box are conserved (Fig. $2 b$ ). Moreover, not all the Streptomyces genes presented a consensus promoter sequence.

The putative promoters $\mathrm{P} 1$ and $\mathrm{P} 2$ were face-to-face and transcription of the two ORFs was divergent from these two promoters. Such a situation has been described for many bacterial and eukaryotic genes (Beck \& Warren, 1988). A similar situation was found recently (Chen et al., 1992) in S. lividans 66, where two ORFs of the Tn4811 transposable element are orientated divergently. Furthermore, the nucleotide sequence of a mercury resistance determinant revealed six ORFs, organized in two divergent units of four and two genes (Sedlmeier \& Altenbuchner, 1992). The two promoters are face-toface. Several other examples of promoters defining complex regulatory regions are known in Streptomyces (Janssen et al., 1989).

Low-resolution S1 nuclease protection experiments revealed the transcription of the two ORFs in the wt strain during the stationary phase. Furthermore, two transcripts of different sizes could be synthesized from ORF2. The amplified NSA6 strain showed no transcription of the ORF2 but increased transcription of ORF1. Further analyses would tell us if these genes were translated and would give us information about the corresponding proteins. Insertional mutagenesis is now being undertaken and could help us to determine the function encoded by these ORFs. AUD6 was shown to hybridize in the two other isolates of $S$. ambofaciens: ATCC 23877 and ETH 11317. Thus, these two ORFs might also exist in these isolates.

How are ORF1 and ORF2 regulated? In the wt strain, the two ORFs were transcribed but amplification of this region led to increased transcription of ORF1 (which might be the regulatory gene) and to no transcription of ORF2. The putative ORF1 gene product might therefore inhibit transcription of ORF2 but not of ORF1. Such an example exists in lysR-lys $A$ genes of $E$. coli (Beck \& Warren, 1988 and references therein), where the LysRdiaminopimelate complex activates $l y s A$ transcription but LysR represses lys $R$ transcription with or without diaminopimelate. In this case, the same binding site might be involved in both effects of the LysR product, and it could imply another supplementary regulatory molecule. In $A U D 6$, the amplification led to the symmetrical situation, where the transcription of the regulatory gene was increased.

We have thus shown that the unstable region contained genes. Recent studies (Leblond et al., 1993) have shown that the unstable region of $S$. lividans corresponds to the 3 o'clock silent region of the $S$. coelicolor chromosome (Kieser et al., 1992). Thus, the unstable region of Streptomyces was expected to contain very few markers. Our study demonstrated the presence of genes in the unstable region of $S$. ambofaciens DSM 40697. Such genes were not detected by classical genetic analyses and perhaps might only be detectable by sequencing these regions. Moreover, these genes could be deleted without affecting significantly the viability of the strains under laboratory conditions. Such a situation has been described for chromosome III of the yeast Saccharomyces cerevisiae on which 182 ORFs for proteins larger than 100 amino acids were detected (Oliver et al., 1992 and references therein). Only a minor fraction of these ORFs $(34 / 182)$ has been identified by classical means and $80 \%$ show no significant homology to any previously sequenced genes. Thus, genome sequencing reveals new functions that have not been discovered by classical techniques.

Furthermore, the deletions and amplifications observed in the mutant strains lead to new DNA sequence associations. It would be interesting therefore to localize the precise deletion end-point in the $1.9 \mathrm{~kb} B a m \mathrm{HI}$ fragment in strain NSA6. Indeed, if this deletion endpoint was localized within ORF1, the new DNA sequence, which originated from the left (in Fig. $1 b$ ) of the AUD6 $1.9 \mathrm{~kb} B a m \mathrm{HI}$ fragment, would become dependent on the P1 promoter. Studies of such deleted mutant strains might therefore allow the detection of potential new genes.

This work was supported by grants from the Ministère de la Recherche et de l'Enseignement Supérieur, and from Université Nancy I. We thank the Ligue Nationale Française Contre le Cancer for its financial support to D.S. We are also grateful to J. L. Souciet for helpful discussions about sequencing techniques. We thank $M$. Guérineau for receiving P.L. in his laboratory to construct the genomic library.

\section{References}

Allison, S. L. \& Phillips, A. T. (1990). Nucleotide sequence of the gene encoding the repressor for the histidine utilization genes of Pseudomonas putida. Journal of Bacteriology 172, 5470-5476. 
AltenbuChNER, J. \& BRÜDERLEIN, M. (1986). The mercury resistance genes of Streptomyces lividans are encoded by an amplifiable DNA sequence. In Book of Abstracts of the 5th International Symposium on the Genetics of Industrial Microorganisms, GIM86, S6-P10.

Altenbuchner, J. \& CUllum, J. (1984). DNA amplification and an unstable arginine gene in Streptomyces lividans 66. Molecular and General Genetics 195, 134-138.

BECK, C. F. \& WARREN, R. A. J. (1988). Divergent promoters, a common form of gene organization. Microbiological Reviews 52 , 318-326.

BibB, M. J., Findlay, P. R. \& Johnson, M. W. (1984). The relationship between base composition and codon usage in bacterial genes and its use for the simple and reliable identification of protein coding sequences. Gene 30, 157-166.

BibB, M. J., JANSSEN, G. R. \& WaRD, J. M. (1985). Cloning and analysis of the promoter region of the erythromycin-resistance gene (ermE) of Streptomyces erythraeus. Gene 38, E357-E368.

BirCh, A., Häusler, A., VöGTLI, M., KREK, W. \& HÜtTer, R. (1989). Extremely large chromosomal deletions are intimately involved in genetic instability and genomic rearrangements in Streptomyces glaucescens. Molecular and General Genetics 217, 447-458.

BOYER, H. W. \& Roulland-Dussoix, D. (1969). A complementation analysis of the restriction and modification of DNA in Escherichia coli. Journal of Molecular Biology 41, 459-472.

BURTON, K. (1956). A study of the conditions and mechanism of the diphenylamine reaction for the colorimetric estimation of deoxyribonucleic acid. Biochemical Journal 62, 315-323.

Chen, C. W., Yu, T. W., Chung, H. M. \& Chou, C. F. (1992). Discovery and characterization of a new transposable element, Tn4811, in Streptomyces lividans 66. Journal of Bacteriology 174, $7762-7769$.

Cullum, J., Altenbuchner, J., Flett, F., Piendl, W. \& Platt, J. (1986). DNA amplification and genetic instability in Streptomyces. Biotechnology and Genetic Engineering Reviews 4, 59-78.

DaNiels, D. L., Schroeder, J. L., Blattner, F. R., Szybalski, W. \& SANGER, F. (1983). A molecular map of coliphage lambda. In Lambda II, pp. 469-517. Edited by R. W. Hendrix, J. W. Roberts, F. W. Stahl \& R. A. Weisberg. Cold Spring Harbor, NY: Cold Spring Harbor Laboratory.

Demuyter, P., Leblond, P., Decaris, B. \& Simonet, J. M. (1988). Characterization of two families of spontaneously amplifiable units of DNA in Streptomyces ambofaciens. Journal of General Microbiology 134, 2001-2007.

DemuYter, P., SchNeIder, D., Leblond, P., Simonet, J. M. \& Decaris, B. (1991). A chromosomal hotspot for multiple rearrangements associated with genetic instability of Streptomyces ambofaciens DSM40697. Journal of General Microbiology 137, 491-499.

DitTrich, W., BETZLER, M. \& SCHREMPF, H. (1991). An amplifiable and deletable chloramphenicol-resistance determinant of Streptomyces lividans 1326 encodes a putative transmembrane protein. Molecular Microbiology 5, 2789-2797.

DoDD, I. B. \& EGAN, J. B. (1987). Systematic method for the detection of potential $\lambda$ Cro-like DNA-binding regions in proteins. Journal of Molecular Biology 194, 557-564.

Feinberg, A. P. \& Vogelstein, B. (1983). A technique for radiolabelling DNA restriction endonuclease fragments to high specific activity. Analytical Biochemistry 132, 6-13.

Fishman, S. E. \& Hershberger, C. L. (1983). Amplified DNA in Streptomyces fradiae. Journal of Bacteriology 155, 459-466.

FleTt, F. \& Cullum, J. (1987). DNA deletions in spontaneous chloramphenicol-sensitive mutants of Streptomyces coelicolor A3(2) and Streptomyces lividans 66. Molecular and General Genetics 207, 499-502.

FUjiTA, Y. \& FUנITA, T. (1987). The gluconate operon gnt of Bacillus subtilis encodes its own transcriptional negative regulator. Proceedings of the National Academy of Sciences of the United States of America 84, 4524-4528.

FujrTA, Y. \& Mrwa, Y. (1989). Identification of an operator sequence for the Bacillus subtilis gnt operon. Journal of Biological Chemistry 264, 4201-4206.

HoHN, B. \& Collins, J. (1980). Small cosmid for efficient cloning of large DNA fragments. Gene 11, 291-298.
Hopwood, D. A., BibB, M. J., Chater, K. F., Kieser, T., Bruton, C. J., Kieser, H. M., Lydiate, D. J., Smith, C. P., WARD, J. M. \& SCHREMPF, H. (1985). Genetic manipulations of Streptomyces-A laboratory manual. Norwich: John Innes Foundation.

Hopwood, D. A., BibB, M. J., Chater, K. F., Janssen, G. R., Malpartida, F. M., SMith, C. P. \& SCHREMPF, H. (1986). Regulation of gene expression in antibiotic-producing Streptomyces. In Regulation of Gene Expression-25 years on. 39th Symposium of Society for General Microbiology, pp. 251-276. Edited by I. Booth \& C. F. Higgins. Cambridge: Cambridge University Press.

Hornemann, U., Otto, C. J., Hoffman, G. G. \& Bertinuson, A. C. (1987). Spectinomycin resistance and associated DNA amplification in Streptomyces achromogenes subsp. rubradiris. Journal of Bacteriology 169, 2360-2366.

HÜTTER, R. (1967). Systematik der Streptomyceten. Basel: Karger Verlag.

HÜTTER, R. \& ECKHARDT, T. (1988). Genetic manipulation. In Actinomycetes in Biotechnology, pp. 89-184. Edited by M. Goodfellow, S. T. Williams \& M. Mordarski. London: Academic Press.

Innis, M. A., Myambo, K. B., Gelfand, D. H. \& Brow, M. A. D. (1988). DNA sequencing with Thermus aquaticus DNA polymerase and direct sequencing of polymerase chain reaction-amplified DNA. Proceedings of the National Academy of Sciences of the United States of America 85, 9436-9440.

JANSSEN, G. R., WARD, J. M. \& BiBB, M. J. (1989). Unusual transcriptional and translational features of the aminoglycoside phosphotransferase gene $(a p h)$ from Streptomyces fradiae. Genes and Development 3, 415-429.

KIESER, H. M., KIESER, T. \& Hopwood, D. A. (1992). A combined genetic and physical map of the Streptomyces coelicolor A3(2) chromosome. Journal of Bacteriology 174, 5496-5507.

KölLING, R. \& LOTHER, H. (1985). AsnC: an autogenously regulated activator of asparagine synthetase A transcription in Escherichia coli. Journal of Bacteriology 164, 310-315.

Leblond, P., Demuyter, P., Moutier, L., LaAkel, M., Decaris, B. \& SIMONET, J. M. (1989). Hypervariability, a new phenomenon of genetic instability, related to DNA amplification in Streptomyces ambofaciens. Journal of Bacteriology 171, 419-423.

Leblond, P., DemuYter, P., Simonet, J. M. \& Decaris, B. (1990). Genetic instability and hypervariability in Streptomyces ambofaciens: towards an understanding of a mechanism of genome plasticity. Molecular Microbiology 4, 707-714.

Leblond, P., Demuyter, P., Simonet, J. M. \& Decaris, B. (1991). Genetic instability and associated genome plasticity in Streptomyces ambofaciens: pulsed-field gel electrophoresis evidence for large DNA alterations in a limited genomic region. Journal of Bacteriology 173, 4229-4233.

Leblond, P., Redenbach, M. \& Cullum, J. (1993). Physical map of the Streptomyces lividans 66 genome and comparison with the related strain Streptomyces coelicolor A3(2). Journal of Bacteriology 175, 3422-3429.

Lipman, D. J. \& Pearson, W. R. (1985). Rapid and sensitive protein similarity searches. Science $227,1435-1441$.

Maniatis, T., Fritsch, E. F. \& SAMBrook, J. (1982). Molecular Cloning: A Laboratory Manual. Cold Spring Harbor, NY: Cold Spring Harbor Laboratory.

OlIVER, S. G. AND OTHERS (1992). The complete DNA sequence of yeast chromosome III. Nature, London 357, 38-46.

PABo, C. O. \& SAUER, R. T. (1984). Protein-DNA recognition. Annual Review of Biochemistry 53, 293-321.

Potekhin, Y. A. \& Danilenko, V. N. (1985). The determinant of kanamycin resistance of Streptomyces rimosus: amplification in the chromosome and reversed genetic instability. Molecular Biology 19, 805-817 (English Translation 19, 672-683).

Pridham, T. G., Anderson, P., Foley, C., Lindenfelser, L. A. \& BENEDICT, R. G. (1956/57). A selection of media for maintenance and taxonomic study of Streptomyces. Antibiotic Annual 1956-1957, 947-953.

SANGer, F., Nicklen, S. \& Coulson, A. R. (1977). DNA sequencing with chain-terminating inhibitors. Proceedings of the National Academy of Sciences of the United States of America 74, 5463-5467. SCHWACHA, A. \& BENDER, R. A. (1990). Nucleotide sequence of the 
gene encoding the repressor for the histidine utilization genes of Klebsiella aerogenes. Journal of Bacteriology 172, 5477-5481.

SedlmeIER, R. \& AltenbuChNER, J. (1992). Cloning and DNA sequence analysis of the mercury resistance genes of Streptomyces lividans. Molecular and General Genetics 236, 76-85.

Short, J. M., Fernandez, J. M., Sorge, J. A. \& Huse, W. D. (1988). $\lambda$ Zap: a bacteriophage $\lambda$ expression vector with in vivo excision properties. Nucleic Acids Research 16, 7583.
SMITH, C. P. \& Chater, K. F. (1988). Structure and regulation of controlling sequences for the Streptomyces coelicolor glycerol operon. Journal of Molecular Biology 204, 569-580.

SOUTHERN, E. M. (1975). Detection of specific sequences among DNA fragments separated by gel electrophoresis. Journal of Molecular Biology 98, 503-517.

Wright, F. \& BiBB, M. J. (1992). Codon usage in the G+C-rich Streptomyces genome. Gene 113, 55-65. 